\title{
Comprehensive Cardiovascular magnetic resonance of myocardial mechanics in mice using three-dimensional cine DENSE
}

\author{
Xiaodong Zhong ${ }^{1}$, Lauren B Gibberman², Bruce S Spottiswoode ${ }^{3}$, Andrew D Gilliam4, Craig H Meyer ${ }^{2,5}$, \\ Brent A French ${ }^{5}$ and Frederick $\mathrm{H}$ Epstein ${ }^{2,5^{*}}$
}

\begin{abstract}
Background: Quantitative noninvasive imaging of myocardial mechanics in mice enables studies of the roles of individual genes in cardiac function. We sought to develop comprehensive three-dimensional methods for imaging myocardial mechanics in mice.

Methods: A 3D cine DENSE pulse sequence was implemented on a 7T small-bore scanner. The sequence used three-point phase cycling for artifact suppression and a stack-of-spirals $k$-space trajectory for efficient data acquisition. A semi-automatic 2D method was adapted for 3D image segmentation, and automated 3D methods to calculate strain, twist, and torsion were employed. A scan protocol that covered the majority of the left ventricle in a scan time of less than 25 minutes was developed, and seven healthy C57BI/6 mice were studied.

Results: Using these methods, multiphase normal and shear strains were measured, as were myocardial twist and torsion. Peak end-systolic values for the normal strains at the mid-ventricular level were $0.29 \pm 0.17,-0.13 \pm 0.03$, and $-0.18 \pm 0.14$ for $E_{r r} E_{c c,}$ and $E_{l l,}$ respectively. Peak end-systolic values for the shear strains were $0.00 \pm 0.08,0.04$ \pm 0.12 , and $0.03 \pm 0.07$ for $E_{r c} E_{r l}$, and $E_{c l,}$ respectively. The peak end-systolic normalized torsion was $5.6 \pm 0.9^{\circ}$.

Conclusions: Using a 3D cine DENSE sequence tailored for cardiac imaging in mice at $7 \mathrm{~T}$, a comprehensive assessment of 3D myocardial mechanics can be achieved with a scan time of less than 25 minutes and an image analysis time of approximately 1 hour.
\end{abstract}

\section{Background}

The application of noninvasive cardiac imaging to genetically-engineered mice is widely used to evaluate the roles of individual genes in cardiac function. These methods have been used to elucidate the roles of genes in normal function and the effects of genes and therapies in cardiac dysfunction [1-5], with the latter typically employing mouse models of myocardial infarction $[6,7]$ or heart failure $[8,9]$. Cardiovascular magnetic resonance (CMR) and echocardiography are both commonly used for these studies $[1,2]$, however CMR is generally considered to be the more accurate modality. Using cine imaging, left ventricular (LV) volume, stroke volume, ejection fraction, and wall thickening can be quantified.

\footnotetext{
* Correspondence: fhe6b@virginia.edu

${ }^{2}$ Radiology Department, University of Virginia, Charlottesville, USA Full list of author information is available at the end of the article
}

In addition, using tissue tracking methods, myocardial strain, twist, and torsion (collectively termed cardiac mechanics) can be measured, which can yield insights into contractile function that are not provided by conventional cine imaging.

A number of different CMR tissue tracking methods may be used to assess cardiac mechanics, including myocardial tagging $[10,11]$, harmonic phase analysis (HARP) [12], velocity encoded phase contrast (PC) $[13,14]$, and displacement encoding with stimulated echoes (DENSE) $[15,16]$. Furthermore, all of these methods are applicable to both humans [10-17] and mice [18-33]. Among these techniques, tagging is the most widely used, but has the significant disadvantage that image analysis is cumbersome and time consuming. HARP simplifies tag analysis, but does so at the expense of reduced spatial resolution. PC imaging can provide
Ciomed Central

() 2011 Zhong et al; licensee BioMed Central Ltd. This is an Open Access article distributed under the terms of the Creative Commons Attribution License (http://creativecommons.org/licenses/by/2.0), which permits unrestricted use, distribution, and reproduction in any medium, provided the original work is properly cited. 
pixel-wise velocity data and direct extraction of velocity values from phase images of the velocity-encoded data. However, extra integral calculation is required to obtain displacement information, and tracking error may accumulate during this procedure [14]. DENSE provides the advantages of high spatial resolution, rapid image analysis, and high displacement accuracy. While some prior tagging studies in mice have been fairly comprehensive in terms of imaging coverage and analysis of threedimensional (3D) mechanics [20,23], prior DENSE studies have not been fully comprehensive in these regards. We recently reported on the development of a cine DENSE method that quantifies 3D mechanics throughout the entire LV [34]. In that report, 3D cine DENSE was applied to imaging humans using a $1.5 \mathrm{~T}$ scanner. In the present study we adapted these methods for imaging mice using a high-field, small-bore scanner, and used them to comprehensively assess the 3D mechanics of the mouse LV.

\section{Methods}

3D cine DENSE Pulse Sequence and Image Reconstruction The 3D cine DENSE method recently developed for imaging the human LV on a $1.5 \mathrm{~T}$ scanner [34] was modified for imaging the mouse LV at a field strength of 7 T. As shown in Figure 1, immediately following Rwave trigger detection, a displacement encoding module consisting of RF and gradient pulses is applied, which stores position-encoded magnetization along the longitudinal axis. The time required to apply the displacement-encoding pulses is $2 \mathrm{~ms}$, which is very short relative to the $R R$ interval, and it can be assumed that no cardiac motion occurs during this period. Also, three-point phase cycling of the second displacementencoding RF pulse is employed for suppression of artifact-generating echoes [35]. The initial displacementencoding module was followed by successive (multiphase) applications of a readout module, which employed an RF excitation pulse, a displacement unencoding gradient, and an interleaved and segmented stack-of-spirals trajectory to sample the 3D $k$-space. A spiral trajectory was chosen to achieve a short echo time (TE) and a time-efficient sampling of $k$-space [36]. Displacement-encoding gradients were designed using the shortest possible time, and, when applicable, unencoding gradients were combined with phase-encoding gradients in the slice-select direction to minimize TE. In addition to ECG triggering, respiratory gating was also used to reduce motion artifact. Multiphase data were acquired throughout at least $90 \%$ of the RR interval, followed by a delay of at least $250 \mathrm{~ms}$ to allow for T1 relaxation. After the delay, the sequence execution resumed after the next detected trigger. Also, a B0 field map was acquired just prior to acquisition of DENSE data to support off-resonance correction during image reconstruction (deblurring). Field map scanning included two 3D acquisitions with different TEs, where each used a single spiral interleave per 3D partition.

DENSE magnitude and phase images were reconstructed online. First, deblurring of the 3D stack-of-spirals dataset was performed as described previously [37]. Briefly, the time signals for both the map and image data were Fourier transformed along the partition direction to form two-dimensional (2D) data sets localized along that direction, and then linear inhomogeneity compensation was performed independently for each slice. Cancellation of interference from artifact-generating echoes and isolation of the displacement-encoded stimulated echo were achieved by combination of the phase cycled datasets as previously described [35]. Subtraction of the background phase was performed to obtain phase images encoded for displacement in the $x$, $y$ and $z$ directions. The corresponding overall magnitude image at each cardiac phase was also calculated using the square root of the sum of the squares of the stimulated echo for all encoding directions.

\section{Computation of Cardiac Mechanics from 3D Cine DENSE Images}

The computation of myocardial strain, twist, and torsion was performed offline in MATLAB (Mathworks Inc., Natick, MA, United States), and included segmentation of the myocardium, phase unwrapping and tissue tracking, and subsequent calculation of specific mechanics parameters. The myocardium was segmented partitionby-partition using a semi-automatic motion-guided segmentation method where manual delineation of the endocardial and epicardial contours is performed at one cardiac phase, and then these contours are automatically propagated to all other cardiac phases using the cardiac displacement data inherently measured by cine DENSE imaging [38]. After segmentation, with the exception of manually identifying the right ventricular insertion point, the valve plane, and the apex, the remainder of the analysis was completely automatic.

For automatic phase unwrapping, the spatiotemporal guided-floodfill algorithm developed for 2D cine DENSE [39] was extended to three spatial dimensions plus time, and was applied to the voxels of the segmented myocardium. The unwrapped phase data were converted to Eulerian displacement by dividing by the displacement encoding frequency, and the 3D Eulerian displacement of each voxel was computed by means of vector addition of the three orthogonal one-dimensional (1D) displacement data.

Next, to convert 3D Eulerian displacements to 3D Lagrangian displacement trajectories, tissue tracking algorithms previously described for 2D data [39] were 


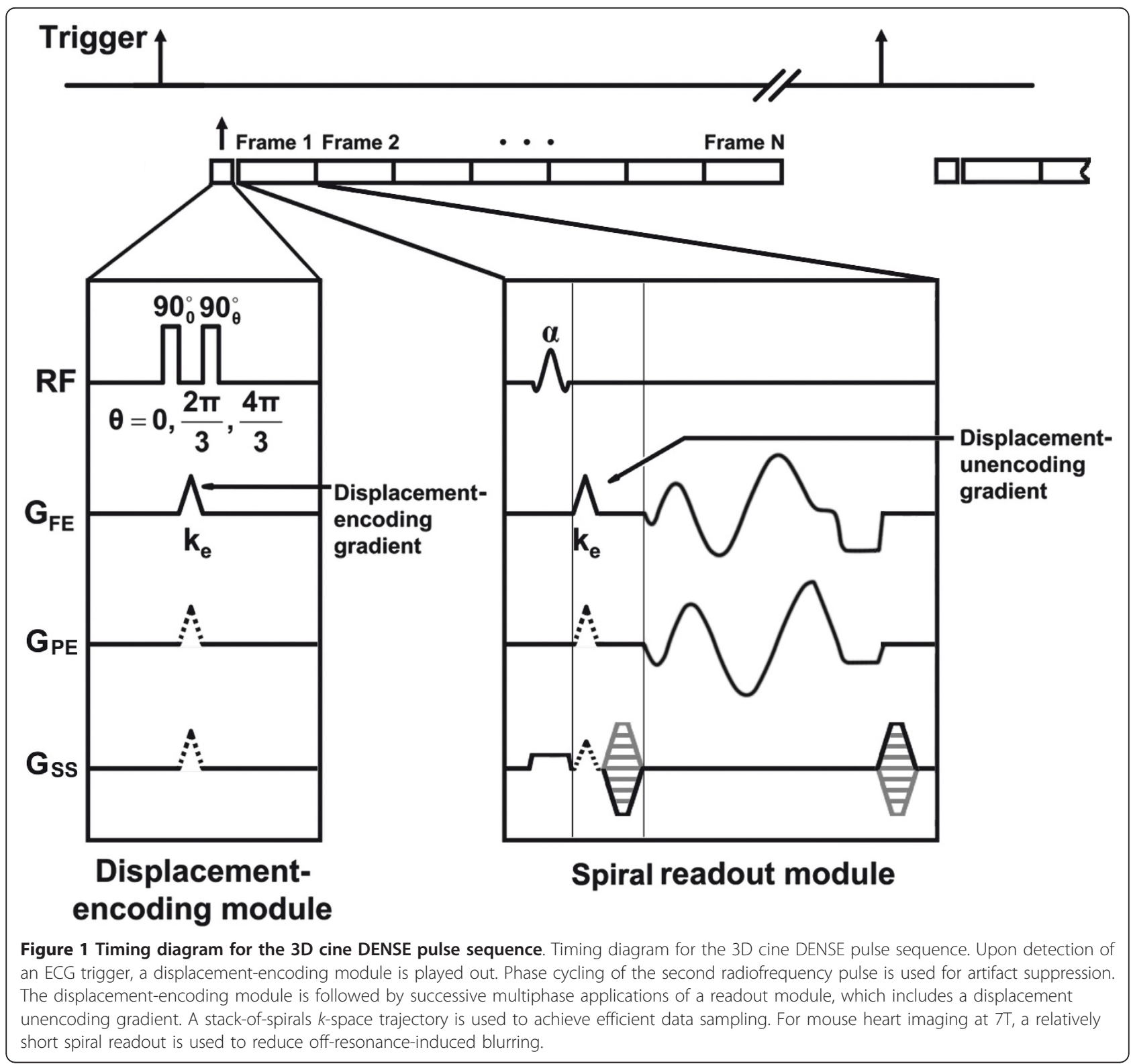

extended to 3D. Scattered data interpolation using radial basis functions of linear splines was performed to calculate the 3D motion trajectories of LV voxel centers identified at the initial acquired cardiac phase [40]. These trajectories were then slightly smoothed using 10th order polynomial functions.

A 3D strain analysis algorithm was also implemented. Specifically, each voxel of interest (VOI) at the initial displacement encoding moment was recorded as a position vector $\overrightarrow{u_{0}}$ in the $n$-dimensional space $R^{n}(n=3$ in this 3D DENSE study):

$$
\overrightarrow{u_{0}}=\left[u_{0,1} \cdots u_{0, n}\right]^{T}
$$

where $u_{0, j}(j=1, \ldots, n)$ corresponded to the coordinate component of $\overrightarrow{u_{0}}$, and the superscript $T$ denotes the matrix transpose operation. $N$ nearest available neighbor voxel position vectors $(N \leq 12)$ were then identified in the closest three partitions within the myocardial contours for this VOI, and denoted as $n$-dimensional vectors $\overrightarrow{p_{0, i}}(i=1, \ldots, N)$. The distance vector matrix $V_{O}$ at the initial displacement encoding moment was formed by assembling the distance vectors pointing from the VOI to its $N$ neighbors, as shown in Equation [2]:

$$
V_{0}=\left[\overrightarrow{q_{0,1}} \cdots \overrightarrow{q_{0, N}}\right]
$$


where $V_{0}$ was an $n \times N$ matrix containing the distance vectors $\overrightarrow{q_{0, i}}$ before deformation (defined in Equation [3]) in columns.

$$
\overrightarrow{q_{0, i}}=\overrightarrow{p_{0, i}}-\overrightarrow{u_{0}} \quad(i=1, \cdots, N)
$$

At any certain cardiac phase $f$, the deformed distance vectors were denoted as $\overrightarrow{q_{f, i}}$, and the corresponding deformed distance vectors are expressed by $V_{f}$ in Equation [4]:

$$
V_{f}=\left[\overrightarrow{q_{f, 1}} \cdots \overrightarrow{q_{f, N}}\right]
$$

Assuming uniform and isotropic strain within the space specified by the VOI and its neighbors, the relationship between $V_{0}$ and $V_{f}$ can be expressed by

$$
V_{f}=F_{f} V_{0}
$$

where $F_{f}$ is the $3 \mathrm{D}$ deformation gradient tensor, an $n \times$ $n$ matrix. In order to obtain a robust solution in the illconditioned case, singular value decomposition (SVD) was used to solve $F_{f}$. Specifically, SVD was performed on $V_{0} V_{0}^{T}$, yielding the diagonal matrix $S$ and unitary matrices $U$ and $V$ as shown in Equation [6]:

$$
V_{0} V_{0}^{T}=U S V^{T}
$$

Then another intermediate matrix $S^{0}$ was calculated as:

$$
\left\{\begin{array}{lr}
S_{i, k}^{0}=S_{i, k} & \text { when } i \neq k \\
S_{i, i}^{0}=1 / S_{i, i} & \text { else }
\end{array}\right.
$$

Finally, the deformation gradient tensor $F_{f}$ was calculated as:

$$
F_{f}=V_{f} V_{0}^{T} V S^{0} U^{T}
$$

and the 3D Lagrangian finite strain tensor $E_{f}$ was calculated as

$$
E_{f}=\left(F_{f}^{T} F_{f}-I\right) / 2
$$

where $I$ is the identity matrix. The strain tensor, $E_{f}$, was decomposed into the local radial, circumferential and longitudinal (RCL) coordinate system, defined with respect to the LV contours at the first cardiac phase [41], to obtain the normal strains $E_{r r}, E_{c c}$, and $E_{l l}$, and the shear strains $E_{r c}, E_{r l}$, and $E_{c l}$ [34]. The definitions of the normal and shear strains are illustrated in Figure 2 .

The twist angle of a voxel was defined as the angle $\theta$ between the radial lines connecting the center of the slice to the center of the voxel of interest at end diastole (or the initial displacement encoding moment) and at each cardiac phase (Figure $2 \mathrm{~A}$ and $2 \mathrm{~B}$ ). A

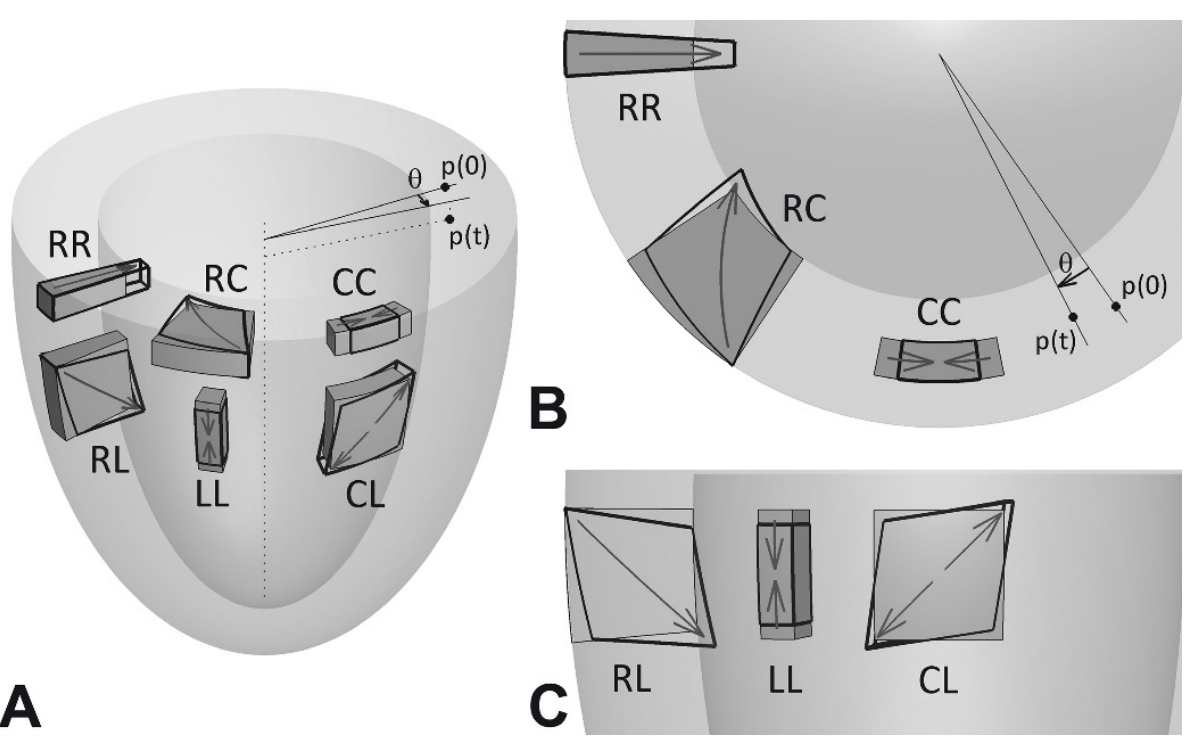

Figure 2 Diagram of left-ventricular strain and twist. Diagram of left-ventricular strain and twist. A 3D view is shown in (A), a 2D short-axis view as observed from the LV base is shown in (B), and a 2D long-axis view is displayed in (C). Strain measures the lengthening or shortening of muscle tissue in a given direction. During contraction, normal tissue lengthens radially (RR), shortens both circumferentially (CC) and longitudinally $(\mathrm{LL})$, and shears slightly $(\mathrm{RC}, \mathrm{RL}, \mathrm{CL})$. Twist $(\theta)$ measures the angular displacement of muscle tissue around the left ventricular centerline. During contraction, normal tissue undergoes a clockwise twist from $p(0)$ to $p(t)$. 
positive angle was defined as a clockwise rotation when viewed from the base of the LV (Figure 2B). Torsion, defined as the normalized gradient of twist in the longitudinal direction, was calculated using Equation [10]:

$$
\text { Torsion }=\frac{\left(\theta_{\text {apex }}-\theta_{\text {base }}\right) R_{L V}}{L_{L V}}
$$

where $\theta_{\text {apex }}$ and $\theta_{\text {base }}$ are the twist angles of the apex and the base of the LV, respectively, $R_{L V}$ is the average short-axis radius of the $\mathrm{LV}$, and $L_{L V}$ is the long-axis length of the LV. This torsion definition is independent of ventricle size, and is well-suited for comparing torsion across mammals of vastly different sizes [42].

\section{Acquisition of 3D Cine DENSE Images in Mice}

Seven wild type (WT) C57BL/6 mice (Jackson Laboratory, Bar Harbor, Maine, USA) were studied under protocols that conformed to the Guide for the Care and Use of Laboratory Animals (NIH publication no. 85-23, revised 1996) and were approved by the Animal Care and Use Committee at the University of Virginia. All imaging was performed on a $7 \mathrm{~T}$ ClinScan CMR system (Bruker, Ettlingen, Germany) equipped with a gradient system that has a maximum strength of $650 \mathrm{mT} / \mathrm{m}$ and a maximum slew rate of $6666.7 \mathrm{mT} \cdot \mathrm{m}^{-1} \cdot \mathrm{ms}^{-1}$. For RF transmission and reception, a birdcage RF coil was used that has a diameter of $32 \mathrm{~mm}$ and an active length of 28 $\mathrm{mm}$. During CMR, the mice were prone within the scanner, and heart rate, respiration, and core body temperature were monitored using a fiber optic, MR compatible system (Small Animal Imaging Inc., Stony Brook, NY, USA). Mouse body temperature was maintained at $36.4 \pm 0.3^{\circ} \mathrm{C}$ during imaging using circulating thermostated water, and anesthesia was maintained using $1.25 \%$ isoflurane in $\mathrm{O}_{2}$ inhaled through a nose cone. Each complete CMR study took approximately 1 hour, including animal preparation, localizer scanning, and functional imaging.

For 3D cine DENSE CMR, pulse sequence parameters included voxel size $=0.25 \times 0.25 \times 0.4 \mathrm{~mm}^{3}, \mathrm{TR}=7$ $\mathrm{ms}, \mathrm{TE}=0.69 \mathrm{~ms}$, flip angle $=15^{\circ}$, number of cardiac phases $=14$, number of averages $=3$, number of spiral interleaves $=27$, number of spiral interleaves per cardiac phase per heartbeat $=1$, and displacement encoding frequency $=1.1 \mathrm{cycles} / \mathrm{mm}$. To achieve a balance of minimal off-resonance-induced blurring and data acquisition efficiency, the spiral readout duration was limited to 3 $\mathrm{ms}$. The specific value of $3 \mathrm{~ms}$ was chosen based on the range of off-resonance frequencies that was recently measured in the mouse heart at a field strength of $7 \mathrm{~T}$ [43]. Specifically, off-resonance frequencies in the range of $\pm 250 \mathrm{~Hz}$ occur under these conditions [43], which is an approximately 5 -fold greater range than in the human heart at $1.5 \mathrm{~T}$ [44]. Since a spiral readout duration of approximately $15 \mathrm{~ms}$ is typically used for human cardiac imaging at $1.5 \mathrm{~T}$ [34], we reasoned that a readout duration reduced by a factor of 5 would provide a reasonable balance between scan efficiency and off-resonance-induced blurring under conditions found in the mouse heart. The field of view was $32 \times 32 \times 8.4 \mathrm{~mm}^{3}$, which covered the entire mouse $\mathrm{LV}$, as the longitudinal dimension of the mouse LV is approximately $6 \mathrm{~mm}$ and the average radius of the mouse LV is approximately 2 $\mathrm{mm}$. The scan time was approximately 23 minutes, depending on heart and respiratory rates. The imaging volume was oriented such that the 3D partition direction was aligned with the long axis of the LV. Fourteen 3D partitions were acquired symmetrically about the $k$ space origin, and zero-padding to 28 partitions was performed during reconstruction. After Fourier Transform in the partition direction, 3 partitions at each end of the volume were discarded to avoid aliasing. The resulting 3D image matrix size was $128 \times 128 \times 22$.

\section{Results}

Image quality was routinely very good in the mid-ventricular region where off-resonance effects are usually minimal. An example demonstrating this image quality is shown in Figure 3, where a magnitude-reconstructed image is displayed as are phase-reconstructed images encoded for in-plane and through-plane displacement. Image blurring was more prevalent mainly toward the apex where off-resonance effects are greater. Nonetheless, image quality was adequate throughout most of the $\mathrm{LV}$ to achieve measurements of tissue displacement and myocardial mechanics. For example, maps of the endsystolic normal strains $E_{r r}, E_{c c}$, and $E_{l l}$ are shown in Figure 4 at basal, mid-ventricular, and apical slices. Figure 5 shows a bar chart of the average end-systolic strains at the basal, mid-ventricular, and apical levels. Heterogeneity in myocardial strains at different levels was observed (Figure 5), which is consistent with prior studies [22-25,32].

Using 3D cine DENSE, in addition to extensive spatial coverage, myocardial mechanics are measured as a function of cardiac phase across the cardiac cycle. For example, data summarizing the time-varying normal strains at the mid-ventricular level for all seven mice are shown in Figure 6, where $E_{r r}, E_{c c}$, and $E_{l l}$, are plotted. These results demonstrate the measurement of normal radial thickening, circumferential shortening, and longitudinal shortening in healthy mice. The high spatial resolution of strain provided by cine DENSE is evident in these measurements, as statistically significant transmural differences in $E_{c c}$ are resolved using this technique, where the thickness of the LV wall is approximately $1 \mathrm{~mm}$. 


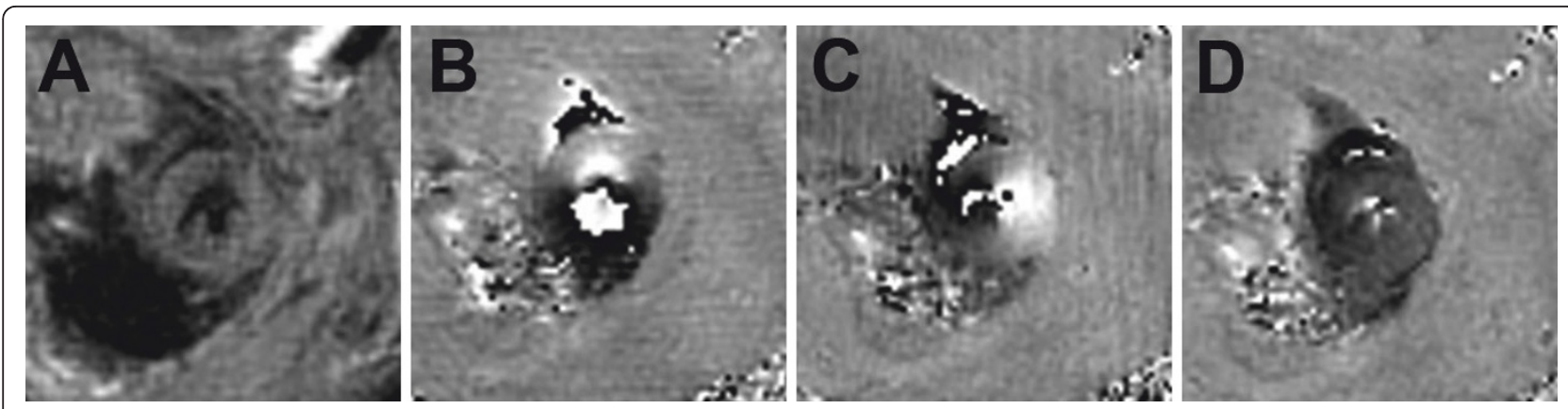

Figure 3 Example end-systolic images from one mid-ventricular short-axis partition. Example end-systolic images from one mid-ventricular short-axis partition from a 3D cine DENSE dataset of a mouse heart. A magnitude-reconstructed image is shown in (A), and phase-reconstructed images are shown in (B-D), where the image in (B) is encoded for displacement in the $y$-direction, the image in (C) is encoded for displacement in the $\mathrm{x}$-direction, and the image in (D) is encoded for displacement in the $z$-direction. Phase wrapping within the myocardium occurred in (B) and $(C)$, and is accounted for by phase unwrapping during image analysis.

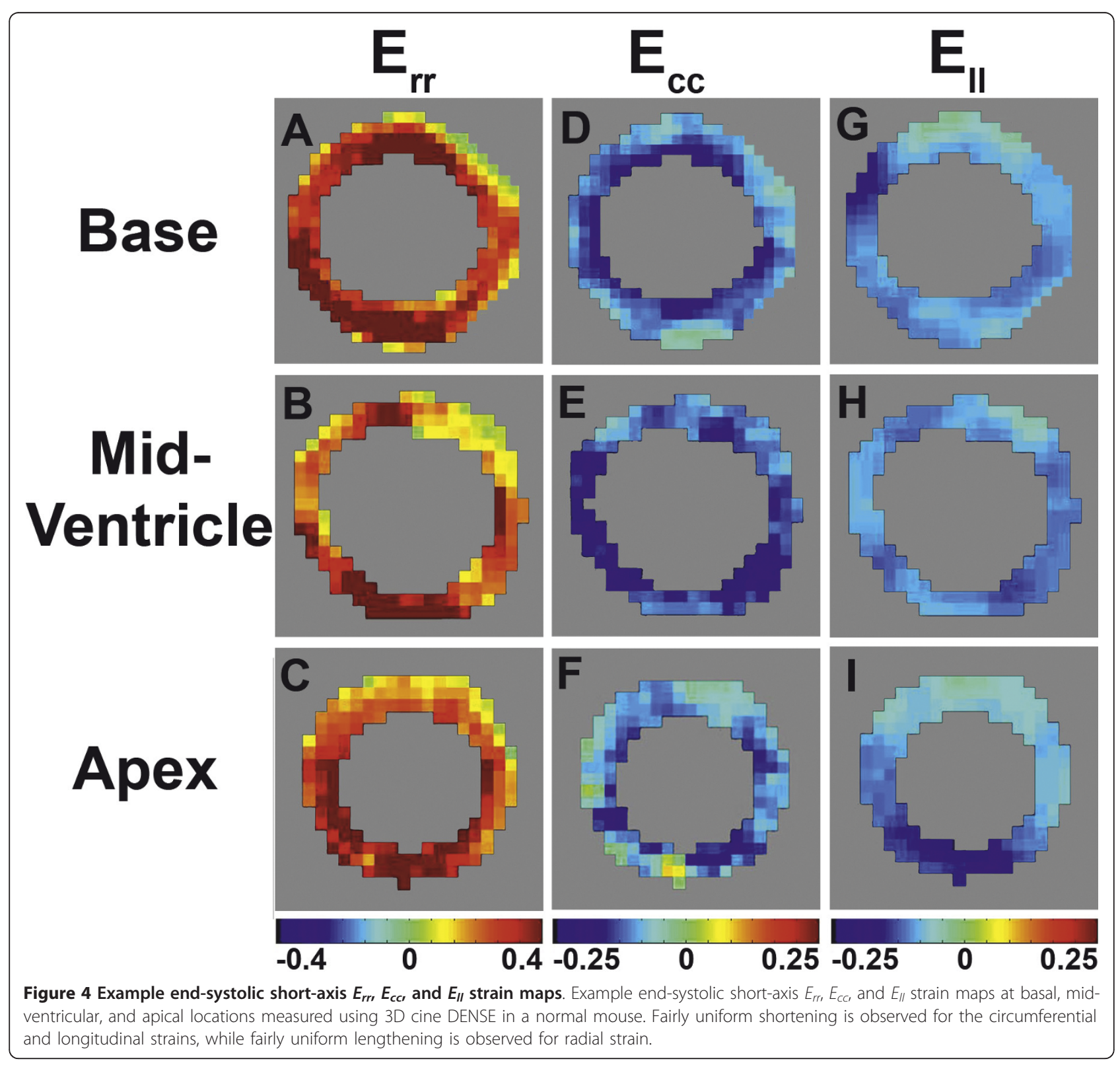




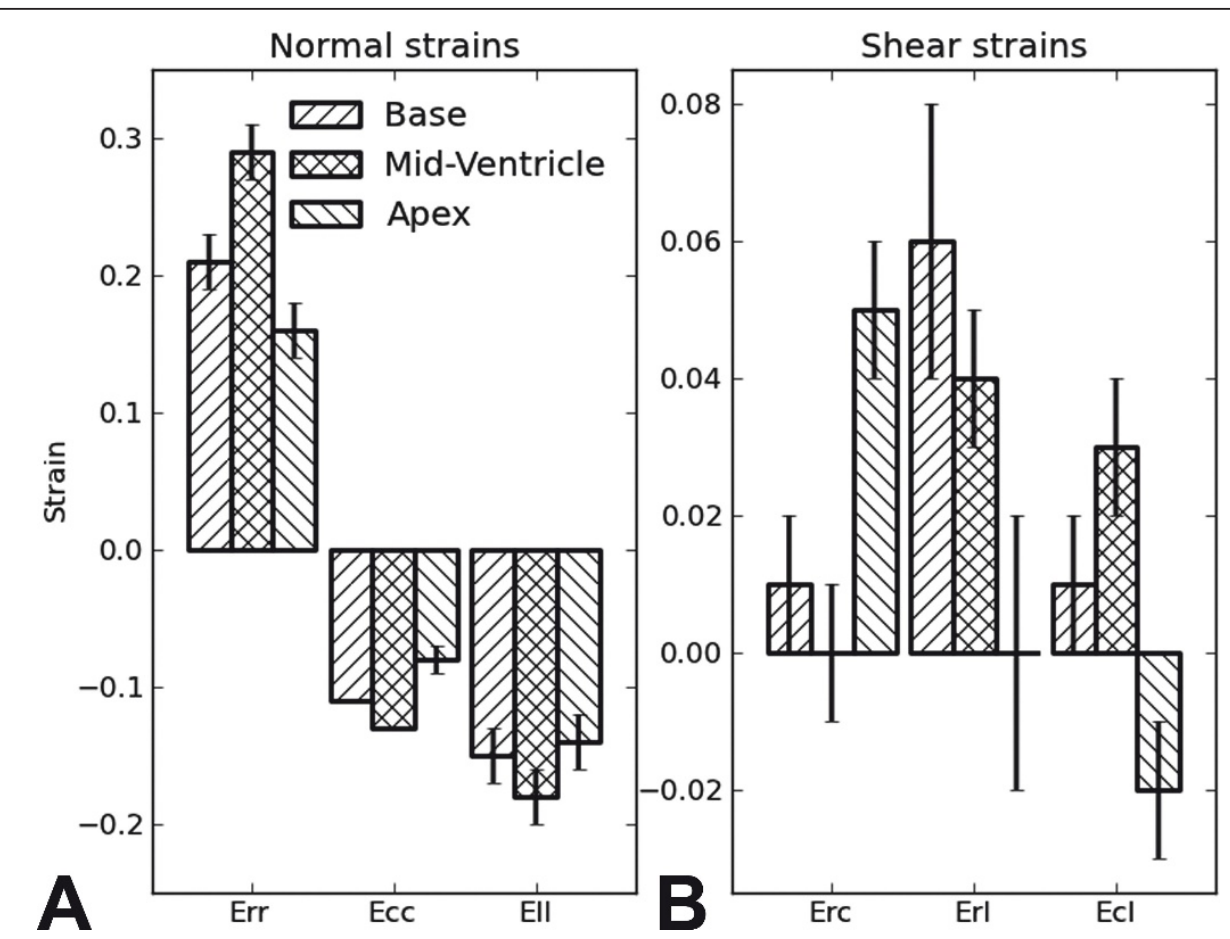

Figure 5 Bar chart of the average end-systolic normal and shear strains. Bar chart of the average end-systolic normal (A) and shear strains (B) at the basal, mid-ventricular, and apical levels for the 7 healthy mice measured by 3D cine DENSE in this study. Data are plotted as mean \pm standard error.

Using 3D cine DENSE, we were also able to measure the shear strains $E_{r c}, E_{r l}$, and $E_{c l}$, as shown for the mid-ventricular region in Figure 7. As in humans, the shear strain magnitudes are lower than the normal strain magnitudes.

Lastly, using displacements that were measured in basal, mid-ventricular, and apical slices, we calculated myocardial twist and torsion as a function of cardiac phase. These results are displayed in Figure 8. Specifically, Figure 8A shows twist as a function of cardiac phase for short-axis slices at three different levels. At the basal level, very little twisting is observed. At the mid-ventricular level, an intermediate amount of counterclockwise twisting is seen, with a peak of approximately $4^{\circ}$ at end systole. At the apical level, a greater amount of counterclockwise twisting is seen, with a peak of approximately $8^{\circ}$ at end systole. In all slices, untwisting is observed during diastole. From twist data measured at multiple longitudinal levels, normalized LV torsion is computed as shown in Figure 8B, with a peak end-systolic value of $5.6 \pm 0.9^{\circ}$.

\section{Discussion}

The major contribution of this study is that a $3 \mathrm{D}$ cine DENSE method is described that enables a

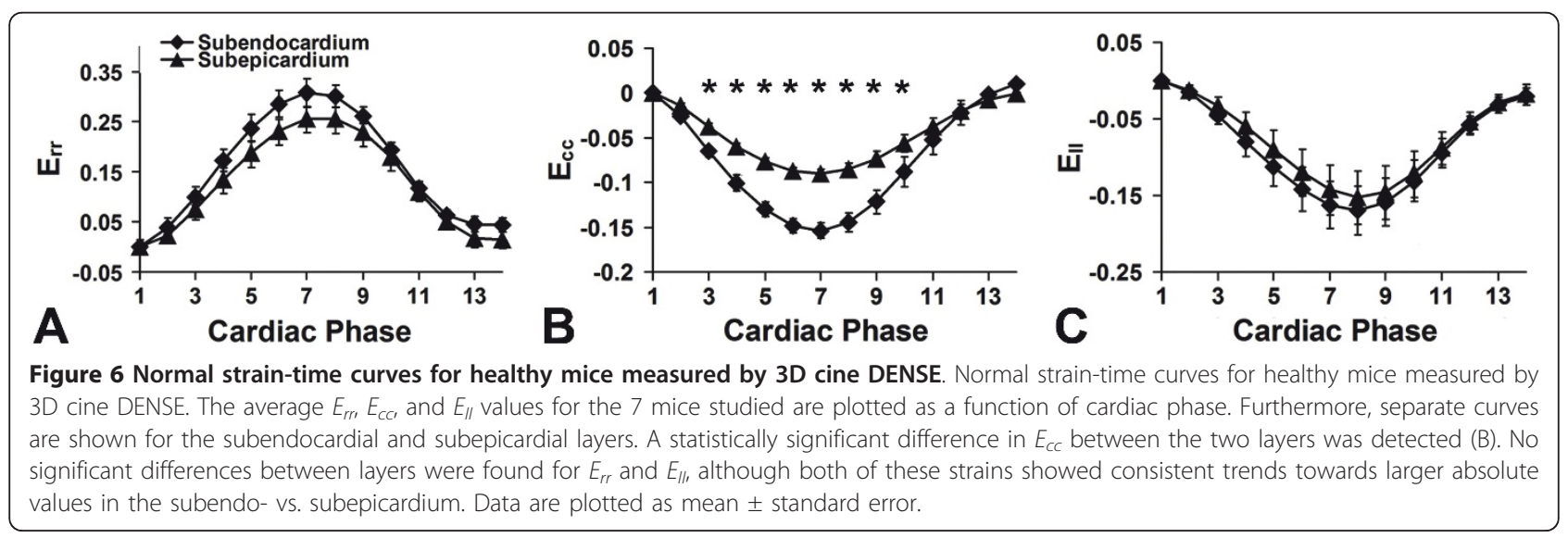




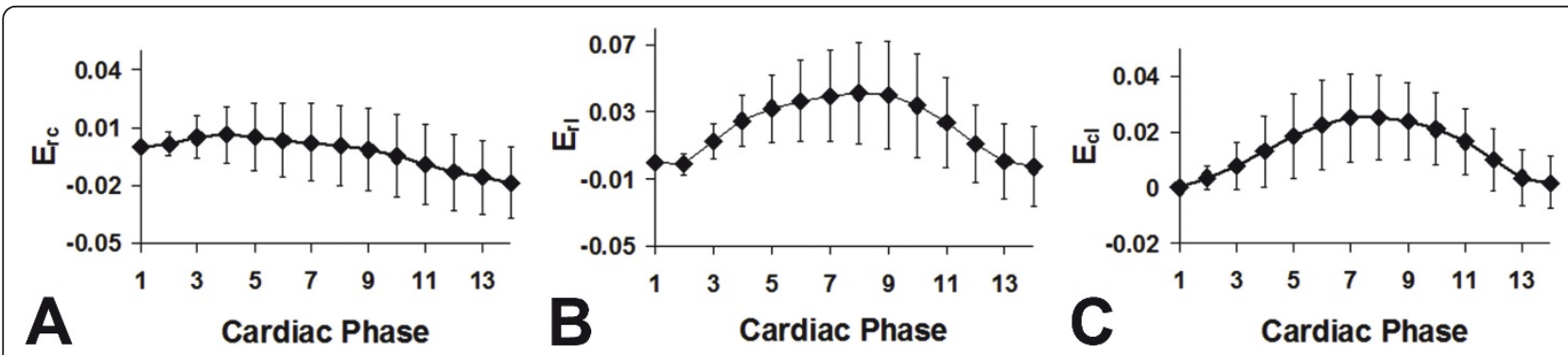

Figure 7 Shear strain-time curves for healthy mice measured by 3D cine DENSE. Shear strain-time curves for healthy mice measured by 3D cine DENSE. The average $E_{r c}, E_{r l}$ and $E_{c l}$ for the 7 mice are plotted as a function of cardiac phase. Data are plotted as mean \pm standard error.

comprehensive evaluation of mouse heart mechanics with a scan time of less than 25 minutes and a data analysis time of approximately one hour. These times are significantly shorter than prior scan times of approximately one hour and prior image analysis times of several hours. The 3D cine DENSE method also provides relatively high spatial resolution, evidenced by the resolution of transmural differences in $E_{c c}$ and by the measurement of shear strains.

We used a 3D stack-of-spirals $k$-space trajectory to achieve efficient data sampling. To avoid excessive blurring due to off-resonance effects at $7 \mathrm{~T}$, we limited the spiral readout duration to $3 \mathrm{~ms}$. We also used 3D field maps and previously described deblurring methods [37]. Using these techniques, image quality was good throughout the central $3.5 \mathrm{~mm}$ of the mouse heart. However, blurring was worse for approximately $1 \mathrm{~mm}$ at the base and $1.5 \mathrm{~mm}$ at the apex, where off-resonance effects were most prominent. In the future, shorter readouts and/or more sophisticated deblurring methods may improve image quality at these locations.

We measured peak normal end-systolic mid-ventricular strains in the mouse heart and found $E_{r r}$ values of $0.29 \pm 0.17, E_{c c}$ values of $-0.13 \pm 0.03$ and $E_{l l}$ values of $-0.18 \pm 0.14$. These values are in close agreement with prior 2D and 3D myocardial tagging studies [20,22-24], 2D HARP studies [25,26], and 2D DENSE studies [31-33]. As shown in Figure 5, we also measured the shear strains in the mouse heart. The systolic values of the shear strains are similar to the results of other shear strain measurements in mice [23]. Also, the shapes of the shear strain curves agree closely with other shear strain measurements in humans $[34,45,46]$. Finally, we computed twist at multiple short-axis levels as well a cardiac torsion. Accounting for different torsion definitions, the twist and torsion values are consistent with those reported previously [21-24].

A limitation of this study is that another independent technique such as myocardial tagging was not used in the same mice to validate 3D cine DENSE measurements. However, the literature contains 3D strain and torsion data for the mouse heart, and our results are similar to the literature values. Also, 3D cine DENSE was directly validated vs. $2 \mathrm{D}$ methods for human imaging [34], and this agreement increases confidence that the 3D cine DENSE data acquisition and analysis methods provide accurate data. Another limitation of the present study is that the image segmentation algorithms that we used were designed for 2D data, and were used separately for each longitudinal partition. A
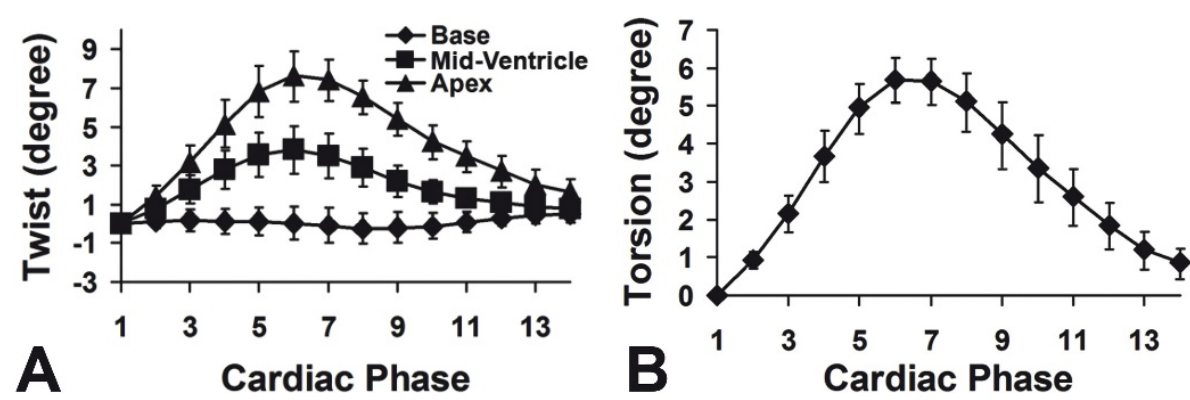

Figure 8 Myocardial twist and torsion as a function of cardiac phase measured by 3D cine DENSE in seven mice. Myocardial twist and torsion as a function of cardiac phase measured by 3D cine DENSE in seven mice. In (A), twist angle as a function of cardiac phase is shown for basal, mid-ventricular, and apical locations. In (B), LV torsion, which is the normalized gradient of twist in the longitudinal direction, is plotted. Data are plotted as mean \pm standard error. 
segmentation algorithm that identifies the 3D endocardial and epicardial surfaces will likely be faster and more accurate.

CMR of myocardial mechanics in mice enables the investigation of the roles of individual genes and experimental therapies in cardiac function. For example, Gilson et al and Vandsburger et al have used myocardial tagging in mice to elucidate the roles of genes that encode for nitric oxide synthases in myocardial mechanics [47,48]. HARP was recently used by Chuang et al to calculate 3D cardiac wall strain distributions from multi-slice tagging data in a genetically engineered mouse model of dilated cardiomyopathy [49]. In addition, PC imaging was applied by Nahrendorf et al to address pathophysiological issues in mice with a combined knockout of the mitochondrial and cytosolic creatine kinase $\left(\mathrm{CK}^{-1-}\right)$ [28]. In a recent study, 2D cine DENSE was also applied to study the role of neuronal nitric oxide synthase in excitation-contraction coupling in the mouse heart [50]. Compared to the other methods, 3D cine DENSE provides the advantages of greater spatial coverage, high spatial and temporal resolution, and rapid post-processing. The 3D cine DENSE technique described in this study has great potential for evaluating the effects of experimental therapies and for quantifying the functional phenotype of genetically-engineered mice.

\section{Conclusions}

Using a 3D cine DENSE sequence tailored for cardiac imaging in mice at 7T, a comprehensive assessment of 3D myocardial mechanics can be achieved with a scan time of less than 25 minutes and an image analysis time of approximately 1 hour.

\section{Acknowledgements}

This study was supported in part by grants NIH NIBIB R01 EB001763 to FHE and NHLBI R01 HL092305 to BAF, and by Siemens Medical Solutions.

\section{Author details \\ ${ }^{1}$ MR R\&D Collaborations, Siemens Healthcare, Atlanta, USA. ${ }^{2}$ Radiology Department, University of Virginia, Charlottesville, USA. ${ }^{3}$ MRC/UCT Medical Imaging Research Unit, University of Cape Town, Cape Town, South Africa. ${ }^{4}$ R\&D Department, A.D. Gilliam Consulting, Providence, USA. ${ }^{5}$ Biomedical Engineering Department, University of Virginia, Charlottesville, USA.}

\section{Authors' contributions}

$\mathrm{XZ}$ and FHE participated in the design and development of the pulse sequence and post-processing software, study design, manuscript drafting and figure preparation. LBG performed data acquisition and analysis, and participated in figure preparation. BSS participated in the development of post-processing software. ADG participated in the development of postprocessing software and figure preparation. CHM contributed to pulse sequence development, providing expertise in spiral imaging. BAF contributed expertise in cardiac physiology and participated in manuscript preparation. All authors read and approved the final manuscript.

\section{Competing interests}

$X Z$ is an employee of Siemens Medical Solutions USA, Inc. FHE received research funding from Siemens Medical Solutions.

Received: 6 July 2011 Accepted: 30 December 2011

Published: 30 December 2011

\section{References}

1. Epstein FH: MR in mouse models of cardiac disease. NMR Biomed 2007, 20:238-255.

2. Collins KA, Korcarz CE, Lang RM: Use of echocardiography for the phenotypic assessment of genetically altered mice. Physiol Genomics 2003, 13(3):227-239.

3. Vandsburger MH, French BA, Helm PA, Roy RJ, Kramer CM, Young AA, Epstein $\mathrm{FH}$ : Multi-parameter in vivo cardiac magnetic resonance imaging demonstrates normal perfusion reserve despite severely attenuated beta-adrenergic functional response in neuronal nitric oxide synthase knockout mice. Eur Heart J 2007, 28:2792-2798.

4. Gilson WD, Epstein FH, Yang Z, Xu Y, Prasad KM, Toufektsian MC, Laubach VE, French BA: Borderzone contractile dysfunction is transiently attenuated and left ventricular structural remodeling is markedly reduced following reperfused myocardial infarction in inducible nitric oxide synthase knockout mice. J Am Coll Cardiol 2007, 50:1799-1807.

5. Yang Z, Bove C, French BA, Epstein FH, Berr SB, Dimaria JM, Gibson J, Carey RA, Kramer CM: Angiotensin II type 2 receptor overexpression preserves left ventricular function after myocardial infarction. Circulation 2002, 106:106-111.

6. Michael LH, Entman ML, Hartley CJ, Youker KA, Zhu J, Hall SR, Hawkins HK Berens K, Ballantyne CM: Myocardial ischemia and reperfusion: a murine model. Am J Physiol 1995, 269:H2147-H2154.

7. Yang Z, Berr SS, Gilson WD, Toufektsian MC, French BA: Simultaneous evaluation of infarct size and cardiac function in intact mice by contrastenhanced cardiac magnetic resonance imaging reveals contractile dysfunction in noninfarcted regions early after myocardial infarction. Circulation 2004, 109:1161-1167.

8. Rockman HA, Wachhorst SP, Mao L, Ross J: ANG II receptor blockade prevents ventricular hypertrophy and ANF gene expression with pressure overload in mice. Am J Physiol 1994, 266: $\mathrm{H} 2468-\mathrm{H} 2475$.

9. Ross J Jr: Dilated cardiomyopathy: concepts derived from gene deficient and transgenic animal models. Circ J 2002, 66:219-224.

10. Zerhouni EA, Parish DM, Rogers WJ, Yang A, Shapiro EP: Human heart: tagging with MR imaging - a method for noninvasive assessment of myocardial motion. Radiology 1988, 169:59-63.

11. Axel L, Dougherty L: MR imaging of motion with spatial modulation of magnetization. Radiology 1989, 171:841-845

12. Osman NF, Kerwin WS, McVeigh ER, Prince JL: Cardiac motion tracking using CINE harmonic phase (HARP) magnetic resonance imaging. Magn Reson Med 1999, 42:1048-1060.

13. van Dijk P: Direct cardiac NMR imaging of heart wall and blood flow velocity. J Comput Assist Tomogr 1984, 8:429-436.

14. Pelc NJ, Noll DC, Pauly JM: Method of noninvasive myocardial motion analysis using bidirectional motion intergration in phase contrast MRI maps of myocardial velocity. US Patent 52576261993.

15. Aletras AH, Ding S, Balaban RS, Wen H: DENSE: displacement encoding with stimulated echoes in cardiac functional MRI. J Magn Reson 1999, 137:247-252

16. Kim D, Gilson WD, Kramer CM, Epstein FH: Myocardial tissue tracking with two-dimensional cine displacement-encoded MRI - development and initial evaluation. Radiology 2004, 230:862-871.

17. Epstein FH: MRI of left ventricular function. J Nucl Cardiol 2007, 14(5):729-744.

18. Henson RE, Song SK, Pastorek JS, Ackerman JJ, Lorenz CH: Left ventricular torsion is equal in mice and humans. Am J Physiol Heart Circ Physiol 2000, 278: $\mathrm{H} 1117-\mathrm{H} 1123$.

19. Epstein FH, Yang Z, Gilson WD, Berr SS, Kramer CM, French BA: MR tagging early after myocardial infarction in mice demonstrates contractile dysfunction in adjacent and remote regions. Magn Reson Med 2002, 48:399-403. 
20. Young AA, French BA, Yang Z, Cowan BR, Gilson WD, Berr SS, Kramer CM, Epstein $\mathrm{FH}$ : Reperfused myocardial infarction in mice: 3D mapping of late gadolinium enhancement and strain. J Cardiovasc Magn Reson 2006, 8:685-692.

21. Zhou R, Pickup S, Glickson JD, Scott CH, Ferrari VA: Assessment of global and regional myocardial function in the mouse using cine and tagged MRI. Magn Reson Med 2003, 49:760-764

22. Liu W, Ashford MW, Chen J, Watkins MP, Williams TA, Wickline SA, Yu X: MR tagging demonstrates quantitative differences in regional ventricular wall motion in mice, rats, and men. Am J Physiol Heart Circ Physiol 2006, 291:H2515-H2521.

23. Zhong J, Liu W, Yu X: Characterization of three-dimensional myocardial deformation in the mouse heart: An MR tagging study. J Magn Reson Imaging 2008, 27:1263-1270.

24. Li W, Liu W, Zhong J, Yu X: Early manifestation of alteration in cardiac function in dystrophin deficient mdx mouse using 3D CMR tagging. J Cardiovasc Magn Reson 2009, 11:40.

25. Zhong J, Liu W, Yu X: Transmural myocardial strain in mouse: quantification of high-resolution MR tagging using harmonic phase (HARP) analysis. Magn Reson Med 2009, 61:1368-1373.

26. Heijman E, Strijkers GJ, Habets J, Janssen B, Nicolay K: Magnetic resonance imaging of regional cardiac function in the mouse. MAGMA 2004, 17:170-178.

27. Streif JU, Herold V, Szimtenings M, Lanz TE, Nahrendorf M, Wiesmann F, Rommel $E$, Haase $A$ : In vivo time-resolved quantitative motion mapping of the murine myocardium with phase contrast MRI. Magn Reson Med 2003, 49:315-321.

28. Nahrendorf M, Streif JU, Hiller KH, Hu K, Nordbeck P, Ritter O, Sosnovik D, Bauer L, Neubauer S, Jakob PM, Ertl G, Spindler M, Bauer WR: Multimodal functional cardiac MRI in creatine kinase-deficient mice reveals subtle abnormalities in myocardial perfusion and mechanics. Am J Physiol Heart Circ Physiol 2006, 290:H2516-H2521.

29. Herold V, Mörchel P, Faber C, Rommel E, Haase A, Jakob PM: In vivo quantitative three-dimensional motion mapping of the murine myocardium with PC-MRI at 17.6 T. Magn Reson Med 2006, 55:1058-1064

30. Dall'armellina E, Jung BA, Lygate CA, Neubauer S, Markl M, Schneider JE: Improved method for quantification of regional cardiac function in mice using phase-contrast MRI. Magn Reson Med 2011.

31. Gilson WD, Yang Z, French BA, Epstein FH: Complementary displacementencoded MRI for contrast-enhanced infarct detection and quantification of myocardial function in mice. Magn Reson Med 2004, 51:744-752.

32. Gilson WD, Yang Z, French BA, Epstein FH: Measurement of myocardial mechanics in mice before and after infarction using multislice displacement-encoded MRI with 3D motion encoding. Am J Physiol Heart Circ Physiol 2005, 288:H1491-H1497.

33. Zhong J, Yu X: Strain and torsion quantification in mouse hearts under dobutamine stimulation using 2D multiphase MR DENSE. Magn Reson Med 2010, 64:1315-1322.

34. Zhong X, Spottiswoode BS, Meyer CH, Kramer MK, Epstein FH: Imaging three-dimensional myocardial mechanics using navigator-gated volumetric spiral cine DENSE MRI. Magn Reson Med 2010, 64:1089-1097.

35. Tsao J, Laurent D: N-SPAMM for efficient displacement-encoded acquisition in myocardial tagging. Proceedings of the 13th Annual Meeting of ISMRM Miami, FL, USA; 2005, 273.

36. Meyer $\mathrm{CH}$ : Spiral echo-planar imaging. In Echo-planar imaging: theory, technique, and application. Edited by: Schmitt F, Stehling MK, Turner R. New York:Springer Verlag; 1998:633-658.

37. Chen W, Meyer CH: Off-resonance correction for 3D imaging using a stack of spirals trajectory. Proceedings of the 14th Annual Meeting of ISMRM Seattle, WA, USA; 2006, 2966.

38. Spottiswoode BS, Zhong X, Lorenz CH, Mayosi B, Meintjes EM, Epstein FH: Motion guided segmentation for cine DENSE MRI. Med Imaging Ana 2009, 13(1):105-115.

39. Spottiswoode BS, Zhong X, Hess AT, Kramer CM, Meintjes EM, Mayosi BM, Epstein FH: Tracking myocardial motion from cine DENSE images using spatiotemporal phase unwrapping and temporal fitting. IEEE Trans Med Imaging 2007, 26(1):15-30.

40. Hardy RL: Theory and applications of the multiquadric-biharmonic method: 20 years of discovery. Comput Math Applic 1990, 19(8/9):163-208.
41. Moore CC, Lugo-Olivieri CH, McVeigh ER, Zerhouni EA: Three-dimensional systolic strain patterns in the normal human left ventricle: characterization with tagged MR imaging. Radiology 2000, 214:453-466

42. Aelen FW, Arts T, Sanders DG, Thelissen GR, Muijtjens AM, Prinzen FW, Reneman RS: Relation between torsion and cross-sectional area change in the human left ventricle. J Biomech 1997, 30:207-212.

43. Beyers RJ, Smith RS, Xu Y, Piras BA, Salerno M, Berr SS, Meyer CH, Kramer CM, French BA, Epstein FH: $\mathrm{T}_{2}$-weighted MRI of post-infarct myocardial edema in mice. Magn Reson Med 2011.

44. Reeder SB, Faranesh AZ, Boxerman JL, McVeigh ER: In vivo measurement of $\mathrm{T}_{2}{ }^{*}$ and field inhomogeneity maps in the human heart at 1.5 T. Magn Reson Med 1998, 39:988-998.

45. Ubbink SWJ, Bovendeerd PHM, Delhaas T, Arts T, van de Vosse FN: Towards model-based analysis of cardiac MR tagging data: relation between left ventricular shear strain and myofiber orientation. Med Image Anal 2006, 10:632-641.

46. Cheng A, Langer F, Nguyen TC, Malinowski M, Ennis DB, Daughters GT, Ingels NB, Miller DC: Transmural left ventricular shear strain alternations adjacent to and remote from infracted myocardium. $J$ Heart Valve Dis 2006, 15(2):209-218.

47. Gilson WD, Epstein FH, Yang Z, Xu Y, Prasad KM, Toufektsian MC, Laubach VE, French BA: Borderzone contractile dysfunction is transiently attenuated and left ventricular structural remodeling is markedly reduced following reperfused myocardial infarction in inducible nitric oxide synthase knockout mice. J Am Coll Cardiol 2007, 50(18):1799-1807.

48. Vandsburger MH, French BA, Helm PA, Roy RJ, Kramer CM, Young AA, Epstein FH: Multi-parameter in vivo cardiac magnetic resonance imaging demonstrates normal perfusion reserve despite severely attenuated beta-adrenergic functional response in neuronal nitric oxide synthase knockout mice. Eur Heart J 2007, 28(22):2792-2798.

49. Chuang JS, Zemljic-Harpf A, Ross RS, Frank LR, McCulloch AD, Omens JH: Determination of three-dimensional ventricular strain distributions in gene-targeted mice using tagged MRI. Magn Reson Med 2010, 64(5):1281-1288

50. Vandsburger $\mathrm{MH}$, French BA, Kramer CM, Zhong X, Epstein FH: Displacement-encoded and manganese-enhanced cardiac MRI reveal that nNOS, not eNOS, plays a dominant role in modulating calcium cycling in the mammalian heart. Am J Physiol Heart Circ Physiol 2011.

\section{doi:10.1186/1532-429X-13-83}

Cite this article as: Zhong et al: Comprehensive Cardiovascular magnetic resonance of myocardial mechanics in mice using threedimensional cine DENSE. Journal of Cardiovascular Magnetic Resonance 2011 13:83.

\section{Submit your next manuscript to BioMed Central and take full advantage of:}

- Convenient online submission

- Thorough peer review

- No space constraints or color figure charges

- Immediate publication on acceptance

- Inclusion in PubMed, CAS, Scopus and Google Scholar

- Research which is freely available for redistribution

Submit your manuscript at www.biomedcentral.com/submit
C Biomed Central 